\title{
Análise de custo do tratamento medicamentoso da artrite reumatóide
}

\author{
Roberta Dyonísio Canaveira Monteiro*,1, Antonio Carlos Zanini²
}

${ }^{1}$ Faculdade de Ciências Farmacêuticas, Universidade de São Paulo, ${ }^{2}$ Faculdade de Medicina, Universidade de São Paulo

*Correspodência:

R. D. C. Monteiro

Rua Artur Prado, 404 - apto 131 -

Bela Vista

01322-0000 - São Paulo - SP, Brasil
Com o objetivo de comparar custos de tratamento para artrite reumatóide com medicamentos modificadores do curso da doença (DMARDs) por um período de 48 meses, foram estudadas cinco diferentes etapas de tratamento fundamentadas em protocolos clínicos recomendados pela Sociedade Brasileira de Reumatologia com cinco ciclos de tratamento. Foi aplicado modelo analítico de decisão baseado na Análise de Markov, considerando as probabilidades do permanecer em algumas destas etapas ou transitar entre elas de acordo com a resposta à terapia. Foram usados os custos diretos com medicamentos, matérias médicohospitalares para sua administração e exames laboratoriais necessários para o monitoramento do paciente. $O$ modelo revelou que o ciclo que representa o uso do metotrexato em monoterapia foi o mais custo/efetivo ( $R \$ 113.900,00$ por paciente em 48 meses), seguido pelo paciente refratário ( $R \$ 1.554 .483,43)$, aquele que utiliza a tripla terapia e depois o biológico ( $R \$ 1.701 .286,76)$, o paciente intolerante ao metotrexato $(R \$ 2.629 .919,14)$, e por fim o resultado daquele que iniciaria o tratamento já com o infliximabe mais metotrexato (R\$ 9.292.879,31). A análise de sensibilidade demonstrou que os resultados encontrados são robustos, mesmo com a variação da eficácia do metrotrexate e do infliximabe.

\author{
Unitermos \\ - Artrite reumatóide/ \\ tratamento medicamentoso \\ - DMARDs \\ - Anti-TNF \\ - Análise farmacoeconômica
}

\section{INTRODUÇÃO}

A reumatologia vem sendo marcada pela introdução de terapias novas e altamente efetivas, mas caras, surgindo à necessidade de correlacionamento entre a sua real necessidade face à probabilidade de bons resultados e custos prováveis. O questionamento cada vez mais recorrente em saúde pública é se os benefícios das inovações medicamentosas compensam seu alto preço (Gabriel, Colyle, Moreland, 2001; Zanini, Wadt, 2006).
A artrite reumatóide, apesar de ser uma doença tratada inicialmente em ambulatório, tem longa duração e alto custo do seu gerenciamento e monitoramento, fazendo o custo do tratamento um importante componente dos provedores de cuidadores de saúde. Diante deste quadro, estimar os custos diretos das diferentes opções de tratamento desta doença é importante ferramenta para os tomadores de decisão (Emery, 2004; Gabriel, Tugwell, Drummond, 2002; Nurmohamed, Dijkmans, 2005; Zanini, Wadt, 2006). 
A farmacoterapia é o alicerce deste tratamento, onde os sintomas devem ser tratados com a combinação de AINEs, corticosteróides e analgésicos narcóticos. Em adição a isso, as drogas modificadoras do curso da doença (DMARDs) são utilizadas para influenciar na progressão da doença, pois estes medicamentos, que variam de agentes antimaláricos, sais de ouro a agentes quimioterápicos, são efetivos em retardar a taxa de progressão radiográfica. Várias combinações de terapias com DMARDs provaram serem mais efetivas que a monoterapia (Gabriel, Tugwell, Drummond, 2002; Lubeck, 2001; Tugwell, 2000).

Até a década de 1980, a filosofia dominante era a da pirâmide, onde o paciente primeiro tratava-se com um medicamento bem tolerado de baixa eficácia enquanto medicamentos mais efetivos, mas mais tóxicos eram administradas tardiamente conforme o curso da doença. Já hoje, o objetivo do tratamento é encontrar uma resposta sustentada ou remissão da doença tão cedo quanto possível dentro do seu processo, antes do início dos danos irreversíveis e perda de função, pois estas conseqüências trazem problemas econômicos e sociais (Polanezyk, Ribeiro, 1998; Scott et al., 1998; Tugwell, 2000).

A evolução tecnológica tenta minimizar estes problemas com a introdução destes novos tratamentos para AR, que incluem DMARDs novos como leflunomida e agentes biológicos (antagonistas de fator de necrose tumoral alfa e antagonista de receptor de interleucinas). Com isso, ocorre um aumento considerável no custo do tratamento da doença nos casos elegíveis, tendendo a necessidade de análises através da farmacoeconomia (Ollendorf et al., 2002; Solomon, Avorn, 2003).

$\mathrm{Na}$ análise farmacoeconômica, aplica-se métodos de avaliação através de uma análise comparativa de alternativas em seu processo (Drummond, et al., 1998; Emery, 2004; Zanini, Wadt, 2006) de ação em termos de ambos os custos e conseqüências. Sob o ponto de vista social, essa análise tem por objetivo estudar a otimização do uso de recursos sem prejuízo da qualidade do tratamento medicamentoso. Na análise, procurou-se considerar a perspectiva do pagador.

$\mathrm{Na}$ estimativa numérica dos custos, alguns fatores econômicos são importantes e devem ser incorporados nas análises, tais como preços de mercado, inflação, depreciação de bens e "valor oportunista". Lembrando que o preço de mercado ou valor cobrado por uma prestação de serviço não reflete, necessariamente o custo real da intervenção, é apenas um de seus componentes (March, Lapsley, 2001; Polanezyk, Ribeiro, 1998)

A percepção do paciente é incorporada na análise de custo-utilidade (ACU), que mensura o custo em relação ao resultado expresso em termos de qualidade de vida, através de utilidade (utility) (Emery, 2004). Na artrite reumatóide, os custos indiretos da inabilidade resultantes da AR são substanciais e muito importantes. Se houver restrição das análises de custo com agentes biológicos e DMARDs somente a custos diretos, os custos com os biológicos são significantemente maiores (Merkesdal et al., 2001; Scott, 2004). Porém, se por um lado os agentes biológicos devem ter efeitos benéficos a médio e longo prazo de uso para o sistema de saúde por reduzir o declínio funcional e concomitantemente diminuição dos custos indiretos, o incremento de custo gerado por esta terapia é substancial e deve ser considerado no momento da escolha.

Apesar destas novas terapias serem promissoras, muitas são mais caras, dificultando o acesso ao tratamento. Assim, as avaliações econômicas dos benefícios dos diversos agentes utilizados para tratá-la são fundametais determinação da alocação ótima de recursos.

\section{MÉTODOS}

Com o objetivo de avaliar e comparar algumas diferenças entre os custos diretos envolvidos foram selecionadas alternativas, a maioria delas baseadas em protocolos clínicos recomendados pela Sociedade Brasileira de Reumatologia (Laurindo et al., 2002). Neste modelo o tratamento é fundamentado na evolução do paciente, que como premissa tem um peso de $70 \mathrm{~kg}$, sem comorbidades, em ciclos de tratamento que podem ser repetidos ou evoluírem por etapas diferentes, onde outros tipos de tratamento são aplicados. Os pacientes podem permanecer em uma determinada etapa ou transitar para outra, de acordo com a resposta ao tratamento ou à evolução da enfermidade, por um horizonte temporal de 48 meses.

Foi aplicado modelo analítico de decisão baseado na Análise de Markov (Gabriel, Tugwell, Drummond, 2002), considerando as probabilidades do permanecer em algumas destas etapas ou transitar entre elas de acordo com a resposta à terapia. Foram usados os custos diretos com medicamentos, materiais médico-hospitalares para sua administração e exames laboratoriais necessários para o monitoramento do paciente.

Os ciclos de tratamento para artrite reumatóide elaborados no modelo, estão de acordo com medicamentos utilizados e tempo de transição entre as etapas. Os medicamentos utilizados foram o ibuprofeno, prednisona, metotrexato, hidroxicloroquina, sulfassalazina, leflunomida e infliximabe, utilizados em associação, conforme os ciclos descritos na Tabela 1 e diagramados na Figura 1.

Foram revisados 93 estudos, dos quais 30 tinham indicadores de eficácia (Monteiro, 2006). Deste, os que apresentavam melhores características para o modelo fo- 
TABELA 1 - Divisão dos ciclos de tratamento para artrite reumatóide elaborados no modelo, de acordo com medicamentos utilizados e tempo de transição entre as etapas

\begin{tabular}{llc}
\hline Ciclo & Medicamentos & Tempo (meses) \\
\hline $1^{\text {o }}$ & Ibuprofeno, prednisona, metotrexato & 48 \\
$2^{\text {o }}$ & Ibuprofeno, prednisona, metotrexato & 3 \\
& Ibuprofeno, prednisona, metotrexato, hidroxicloroquina, sulfassalazina & 6 \\
& metotrexato, infliximabe & 39 \\
$3^{\text {o }}$ & Ibuprofeno, prednisona, metotrexato & 3 \\
& Ibuprofeno, prednisona, metotrexato, hidroxicloroquina, sulfassalazina & 6 \\
& metotrexato, leflunomida & 6 \\
& metotrexato, infliximabe & 33 \\
$4^{\text {o }}$ & Ibuprofeno, prednisona, metotrexato & 3 \\
& Leflunomida & 6 \\
$5^{\text {o }}$ & metotrexato, infliximabe & 39 \\
\hline
\end{tabular}

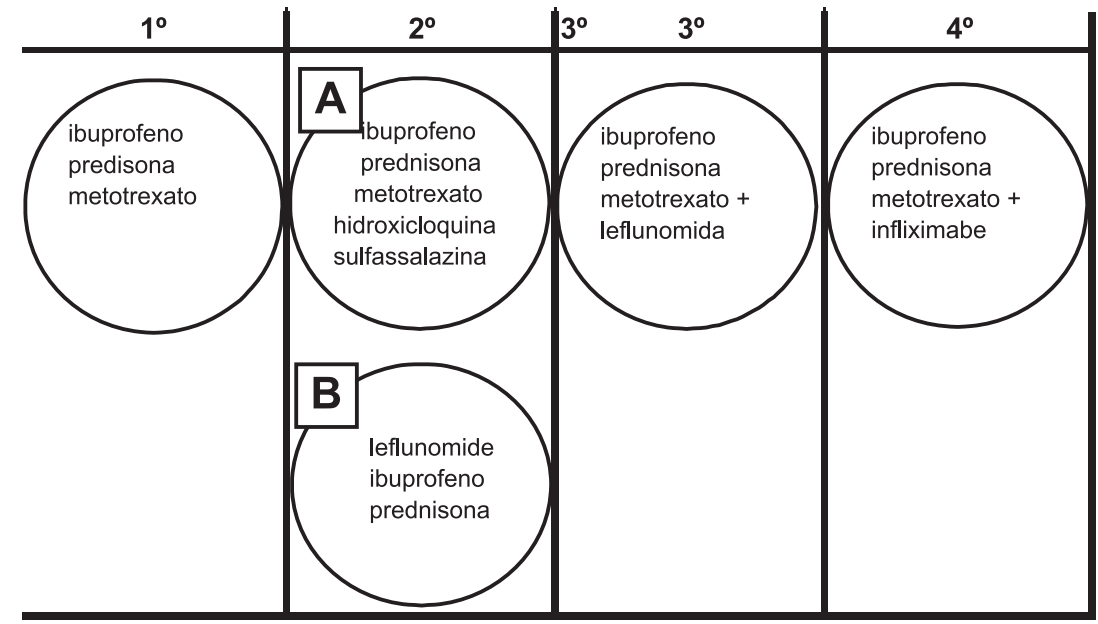

FIGURA 1 - Etapas da doença e medicamentos utilizados em cada uma delas baseado nas alternativas disponíveis no Consenso.

ram dois artigos de revisão publicados na literatura médica, Therapeutic Strategies for Rheumatoid Arthritis (2004), e Efficacy, Tolerability and Cost Effectiveness of DiseaseModifying Antirheumatic Drugs and Biologic Agents in Rheumatoid Arthritis (2005).

O indicador de eficácia utilizado para este modelo foi o índice de ACR20 (critério do American College of Rheumatology) atingido por paciente.

As Figuras 2 a 6, abaixo, representam os diagramas dos ciclos de tratamento da artrite reumatóide estudados neste modelo.

Para estimativa de custos de tratamento, considerouse o seguinte:

- os custos direitos foram estimados segundo alterna-

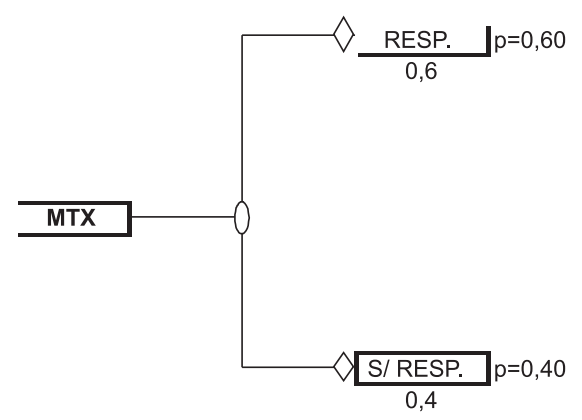

FIGURA 2 - Opções e probabilidade do paciente respondedor ao metotrexato submetido às etapas de tratamento do Ciclo 1 . (Nurmohamed, Dijkamans, 2005). RESP.= paciente que atinge $20 \%$ de melhora no critério do ACR; S/ RESP.= paciente que não atinge $20 \%$ de melhora no critério do ACR; MTX $=$ metotrexato; $\mathrm{p}=$ porcentagem de pacientes que atingem ou não resposta. 


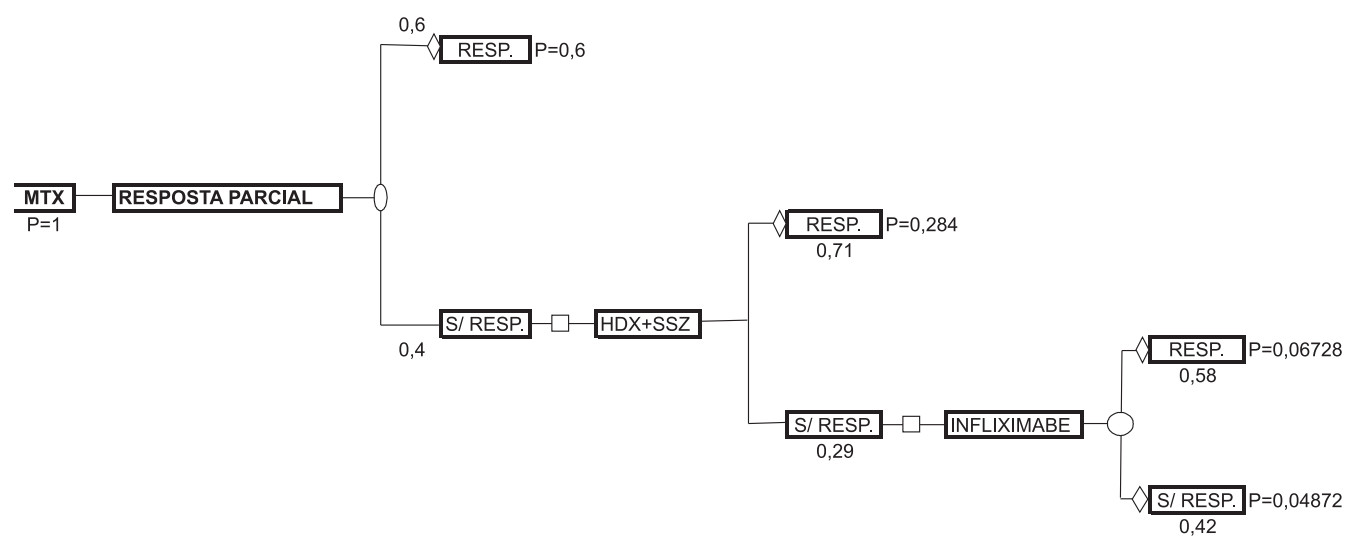

FIGURA 3 - Opções e probabilidade de resposta parcial ao metotrexato submetido às etapas de tratamento do Ciclo 2 (Nurmohamed, Dijkamans, 2005; O'Dell, 2004). RESP.= paciente que atinge 20\% de melhora no critério do ACR; S/ RESP.= paciente que não atinge $20 \%$ de melhora no critério do ACR; MTX = metotrexato; SSZ = sulfassalazina; HDX $=$ hidroxicloroquina; $\mathrm{p}=$ porcentagem de pacientes que atingem ou não resposta.

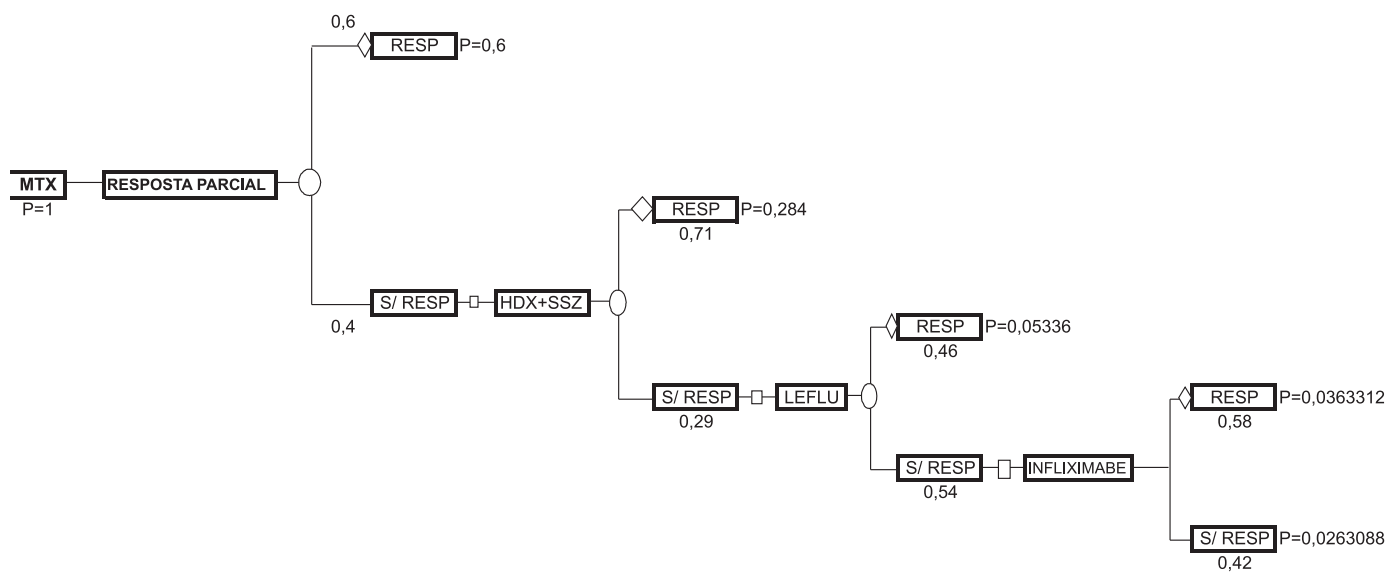

FIGURA 4 - Opções e probabilidade do paciente refratário submetido às etapas de tratamento do Ciclo 3. (Nurmohamed, Dijkamans, 2005; O’Dell, 2004). RESP.= paciente que atinge 20\% de melhora no critério do ACR; S/ RESP.= paciente que não atinge $20 \%$ de melhora no critério do ACR; MTX = metotrexato; SSZ = sulfassalazina; HDX = hidroxicloroquina; LEFLU = leflunomida; $p=$ porcentagem de pacientes que atingem ou não resposta.

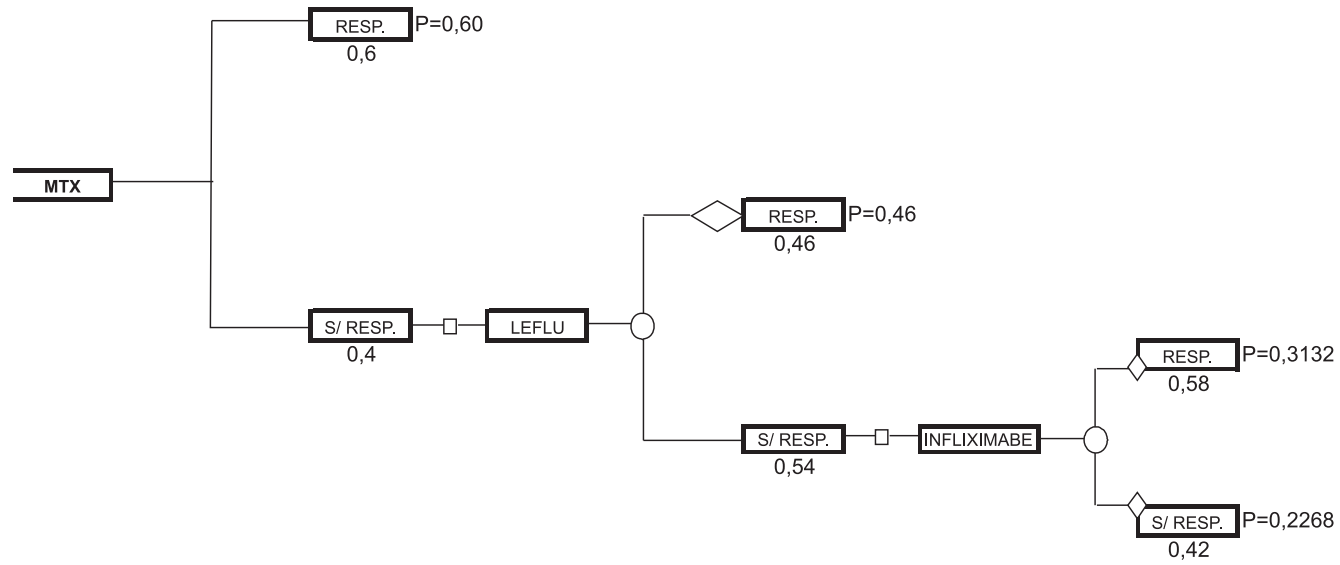

FIGURA 5 - Opções e probabilidade do paciente intolerante ao metotrexato submetido às etapas de tratamento do Ciclo 4. (Nurmohamed, Dijkamans, 2005; O’Dell, 2004). RESP.= paciente que atinge 20\% de melhora no critério do ACR; S/ RESP.= paciente que não atinge $20 \%$ de melhora no critério do ACR; MTX = metotrexato; SSZ = sulfassalazina; HDX = hidroxicloroquina; LEFLU = leflunomida; $\mathrm{p}=$ porcentagem de pacientes que atingem ou não resposta. 


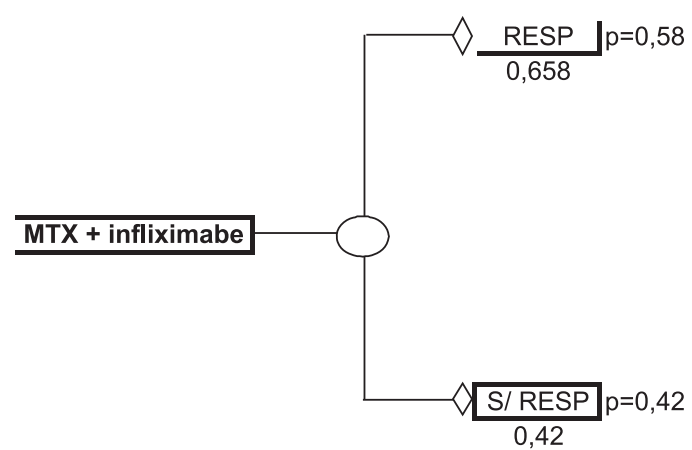

FIGURA 6 - Opções e probabilidade do paciente que inicia a terapia com o biológico submetido às etapas de tratamento do Ciclo 5. (Nurmohamed, Dijkamans, 2005; O'Dell, 2004). RESP.= paciente que atinge $20 \%$ de melhora no critério do ACR; S/ RESP.= paciente que não atinge $20 \%$ de melhora no critério do ACR; MTX = metotrexato; $\mathrm{p}=$ porcentagem de pacientes que atingem ou não resposta.

tivas do Consenso Brasileiro para o Diagnóstico e Tratamento da Artrite Reumatóide (Laurindo et al., 2002), com medicamentos utilizados para o tratamento da artrite reumatóide, materiais médico-hospitalares envolvidos na administração destes medicamentos, e exames laboratoriais oriundos do uso destes medicamentos;

- os resultados econômicos foram expressos em custo total de tratamento por paciente para cada ciclo, após 48 meses;

- os preços dos medicamentos e materiais foram baseados em preço de venda dos medicamentos de referência, retirados da Revista Brasíndice (GUIA Farmacêutico Brasíndice, 2006), Janeiro de 2006, preço máximo ao consumidor, $18 \%$.

- os custos dos exames realizados para o monitoramento dos efeitos dos DMARDs foram baseados na Tabela de Honorários Médicos da Associação Médica Brasileira (CLASSIFICAÇÃO Brasileira Hierarquizada de Procedimentos Médicos, 2005), CH de R\$ 0,27.

Inicialmente, foi calculada a dose diária dos medicamentos recomendada pelo Consenso, considerando as doses médias preconizadas em um paciente de $70 \mathrm{~kg}$ e os medicamentos baseados na sua nomenclatura segundo as Denominações Comuns Brasileiras (DCB).

Os preços dos produtos farmacêuticos foram retirados do periódico Brasíndice e transformados matematicamente para identificar o preço por unidade $(\mathrm{mg})$ para cada medicamento; a seguir, aplicando-se as quantidades por dose preconizadas pelo Consenso, obteve-se o custo por dose de medicamento e por etapa de tratamento.

Por fim, o custo dos ciclos de tratamento foi obtido pela somatória dos custos das etapas de tratamento envolvidas em cada um deles, obedecendo ao intervalo de tempo determinado como premissa no modelo e as probabilidades de transição entre eles, até chegarmos ao horizonte de 48 meses.

\section{RESULTADOS}

O custo das etapas de tratamento foram de $\mathrm{R} \$ 683,40$ (Etapa 1), R\$ 2.788,44 (Etapa 2), R\$ 3.262,65 (Etapa 3), $\mathrm{R} \$ 3.839,25$ (Etapa 4), R \$ 53.898,70 (Etapa 5).

O custo dos ciclos de tratamento estão apresentados na figura abaixo.

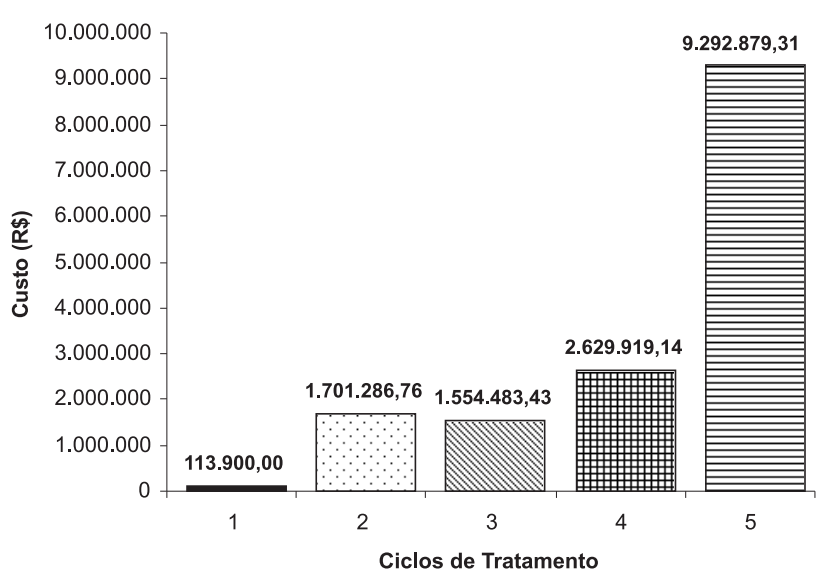

FIGURA 7 - Relação custo/efetividade entre as alternativas de ciclos de tratamento utilizadas no modelo, por índice de ACR 20 atingido.

Foi aplicada uma análise de sensibilidade, procedimento analítico pelo qual se avalia a solidez dos resultados encontrados no estudo, por intermédio da mudança de variáveis chave dentro do modelo. Neste caso, foi feita a variação da porcentagem de eficácia do metotrexato, utilizando os valores de resposta para chegar ao índice de ACR 20 de 45,50 e $55 \%$, por esta variável ser de grande importância no estudo, e também a variação da eficácia do infliximabe para $62 \%$, quando o metotrexato tem $45 \%$. Os valores estão representados nas Figuras 8 a 10.

A Figura 11 mostra o impacto da efetividade/eficácia do metotrexato dentro dos ciclos de tratamento.

\section{DISCUSSÃO}

Diante das numerosas variáveis que envolvem o tratamento de um paciente com artrite reumatóide procurouse comparar as probabilidades de eficácia e custos de diversos protocolos de tratamento, buscando considerar a possibilidade de que agentes biológicos tenham efeitos benéfi- 


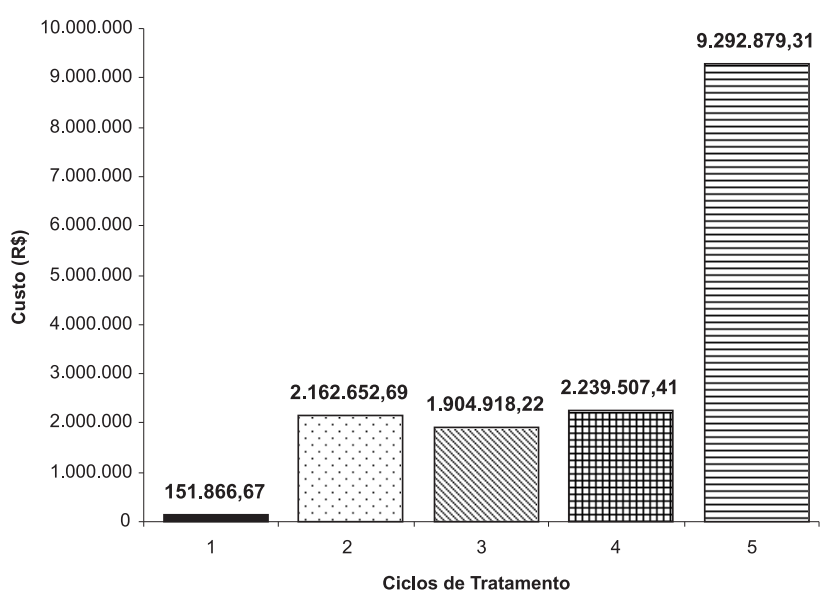

FIGURA 8 - Comparação dos custos dos ciclos de tratamento com o indicador de efetividade de índice de ACR 20 atingido pelo metotrexato de $45 \%$.

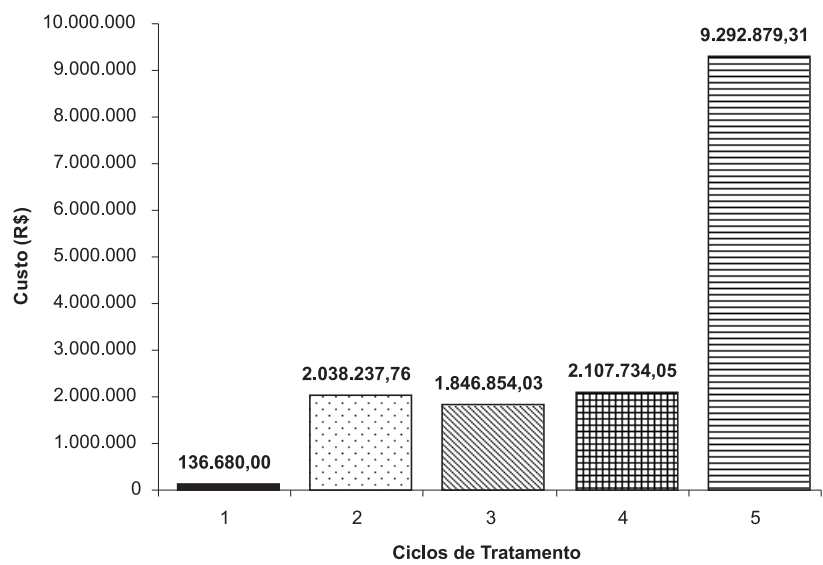

FIGURA 9 - Comparação dos custos dos ciclos de tratamento. com o indicador de efetividade de índice de ACR 20 atingido pelo metotrexato de 55\%.

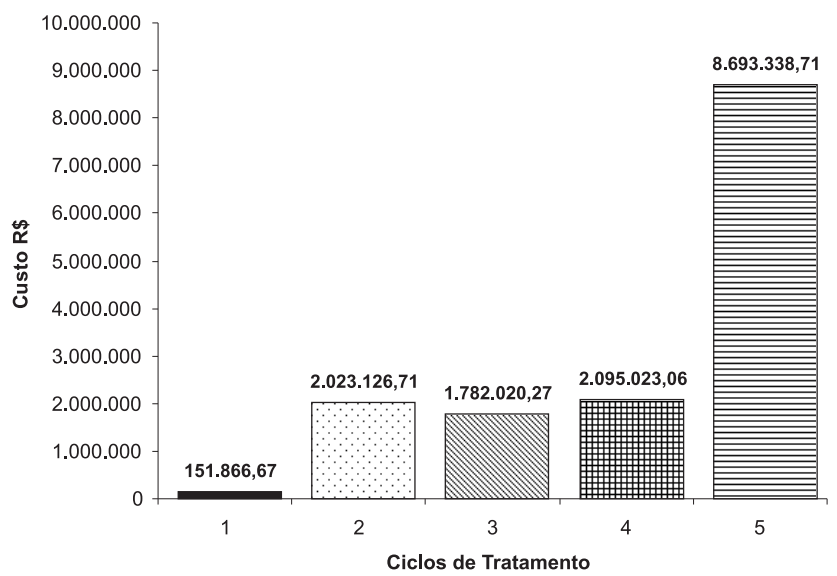

FIGURA 10 - Comparação dos custos dos ciclos de tratamento. com o indicador de efetividade de índice de ACR 20 atingido pelo metotrexato de $45 \%$ e infliximabe $62 \%$.

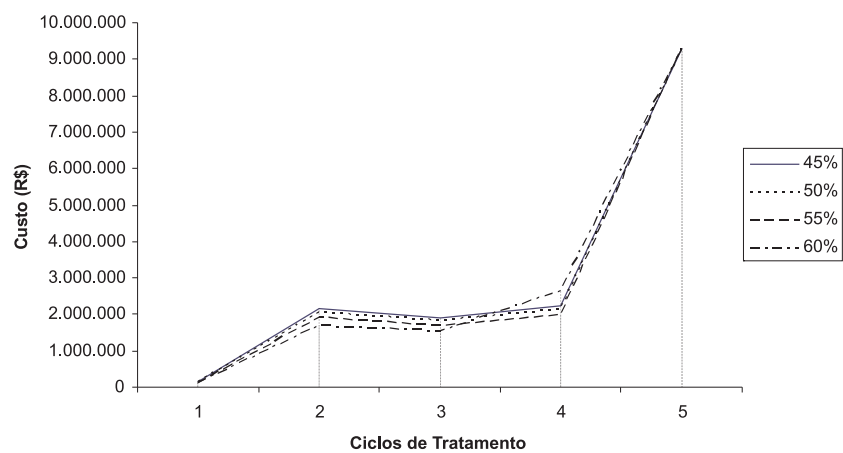

FIGURA 11 - Comparação dos custos dos 5 ciclos de tratamento segundo variação de porcentagem de efetividade/ eficácia do metotrexato.

cos a médio e em longo prazo com a diminuição dos custos indiretos, relacionados principalmente com a perda de produtividade. Apesar da diferença de preços, o seu uso poderia reduzir estes custos para o sistema de saúde e assim provar o seu benefício e sua eficiência para os pacientes e para os tomadores de decisão em saúde.

Com o objetivo de quantificar ao menos parte deste impacto econômico para a sociedade, foi desenvolvido um modelo econômico que seguiu os princípios da Análise de Markov (Gabriel, Tugwell, Drummond, 2002), baseando-se em revisão de literatura e considerando os custos diretos dos medicamentos, dos materiais médico-hospitalares utilizados em sua administração, e dos exames laboratoriais necessários para o monitoramento pelo uso das drogas modificadoras do curso da doença, bem como a eficácia destas drogas modificadoras do curso da doença, retiradas de artigos de revisão.

Na revisão de literatura (Monteiro, 2006), observouse à dificuldade de encontrar evidências comparativas de eficácia ou efetividade para o tratamento da artrite reumatóide. Além da diversidade de indicadores utilizados para mensurar este fim e, além disso, existem diversos tipos de pacientes com difícil harmonização de quadro para que pudessem enquadrados na análise.

Apesar destes dados de eficácia não significarem a certeza que um nível específico de resposta de um estudo possa ser diretamente comparado a outro estudo, pela falta de estudos frente à frente (head-to-head) entre as terapias escolhidas, eles foram utilizados como indicadores de efetividade. Isto porque não existirem ainda, no Brasil, dados suficientes que nos permitam analisar aspectos farmacoeconômicos relevantes no caso da artrite reumatóide.

Este fato resulta em um viés, pois não se podem inferir com segurança se estes resultados de eficácia seriam os mesmos se o paciente iniciasse o tratamento em qualquer 
um dos ciclos, e fosse nele até o fim. A dúvida fica na eficácia dos DMARDs depois de já ter sido utilizado outro DMARD antes dele.

As variáveis utilizadas no modelo, medicamentos, materiais e exames, foram catalogadas e seus preços foram retirados de revistas utilizadas para este fim. Desta forma houve a possibilidade da diferenciação do seu preço de aquisição para o custo total do tratamento medicamentoso, que, no caso, não envolve somente o preço dos medicamentos, mas a posologia de cada um deles e também o consumo ou não de materiais médico hospitalares na sua administração e a necessidade ou não de exames laboratoriais.

O cálculo de custo destas etapas obedeceu ao tempo determinado pelo Consenso (Laurindo et al., 2002) para cada uma delas, chegando ao valor de $\mathrm{R} \$ 683,40, \mathrm{R} \$ 2.788,44, \mathrm{R} \$$ $3.262,65, \mathrm{R} \$ 3.839,25$ e $\mathrm{R} \$ 53.898,70$ por paciente, para as etapas 1, 2a , 2B, 3 e 4, respectivamente. Este resultado demonstrou uma progressão de custo já esperada, de acordo com a substituição dos DMARDs mais antigos pelos mais novos, que tem um maior preço de aquisição.

Além do peso econômico gerado por estes medicamentos, a necessidade de monitoramento devido a seu uso também deve ser levada em consideração e não segue a mesma proporção. As etapas 1, 2 A e 3 tiveram 38, 32 e $27 \%$ de seu total de custo representada por este monitoramento; já nas etapas $2 \mathrm{~B}$, onde o leflunomide é utilizado sem à combinação com o MTX, e 4, onde o infliximabe é utilizado em combinação com o metotrexato, este custo representou apenas 16 e 1\%.

Apesar da possibilidade de desvios, tanto pela escassez de dados na literatura como por não estar sendo levada em consideração parte importante dos custos diretos como os eventos adversos oriundos de cada classe de medicamentos, e tão pouco os custos indiretos que são confirmados como os custos que mais impactam nos pacientes com artrite reumatóide por esta ser uma doença incapacitante, foi possível comparar o custo de cada ciclo elaborado e o impacto gerado pelo incremento de custo durante a progressão do paciente em cada um destes ciclos.

Entre os cinco ciclos elaborados, os quatro primeiros foram desenvolvidos para refletir situações clínicas já existentes. O primeiro ciclo é fundamentado naquele paciente que responde bem à terapia inicial com metotrexato e por isso se mantém nele durante os 48 meses do estudo. Este ciclo resultou na razão de custo/eficácia mais baixa entre todas as outras alternativas estudadas, ou seja, o menor valor para um paciente atingir o índice de ACR20.

Este custo é importante, pois as atuais diretrizes de tratamento são a favor da detecção da doença com um diagnóstico precoce, com início da terapia, em tempo adequado com os medicamentos que possam retardar a progressão da destruição articular, e assim, a incapacidade do paciente.

$\mathrm{Na}$ avaliação de intervenções utilizadas na promoção da saúde, além da segurança, da eficácia ("a intervenção funciona em condições ideais?"), da efetividade ("a intervenção funciona nas condições do dia-a-dia?") deve-se acrescentar a questão da eficiência. A essência deste elemento é avaliada através da pergunta: "Estamos obtendo o melhor benefício pelos recursos que estamos utilizando"?

O tomador de decisão seja ele o prescritor ou as entidades governamentais, devem ter em mente a eficiência na alocação dos seus recursos, pois para os dois maiores pagadores de serviços médicos no Brasil, o Sistema Único de Saúde e os planos de saúde, os custos relacionados à saúde têm atingido patamares insustentáveis, havendo a necessidade de otimização dos recursos.

Se as análises farmacoeconômicas relacionadas a esta doença forem restritas somente a custos diretos, como foi o caso do estudo e considerando as limitações já citadas, a razão de custo/eficácia do tratamento com os agentes biológicos são significantemente maiores que os outros medicamentos disponíveis para o tratamento da doença.

O estudo dos custos diretos das diferentes opções de tratamento da artrite reumatóide, através do desenvolvimento de um modelo analítico baseado em dados da literatura científica, mostrou que os trabalhos publicados ainda não trazem segurança quanto à probabilidade de sucesso do tratamento. Apesar das dificuldades, foi possível construir uma árvore decisão (conforme o modelo de Markov), com base nos dados publicados.

O modelo de análise farmacoeconômica com custos e desfechos de tratamento utilizados para a artrite reumatóide permitiu concluir que o preço de medicamentos mais recentes, como biológico influximabe e o lefluonomida dos medicamentos com alto preço, impacta de modo relevante, elevando o custo geral do tratamento em relação aos demais fármacos habitualmente utilizados, principalmente quando utilizados como terapia inicial.

Permitiu verificar também que o custo inerente ao monitoramento necessário quando do uso dos medicamentos tem maior impacto no custo total do tratamento quando relacionados aos de menor preço, ao contrário dos medicamentos com alto preço.

Mesmo quando um paciente não responda bem ao metotrexato utilizado nas fases iniciais, ou seja, intolerante a este medicamento, os custos incrementais demonstram que a tripla terapia (metotrexato, sulfassalazina e hidroxicloroquina) devem ser tentados antes dos medicamentos de maior custo (influxibame e leflunomida).

Se o paciente que é tratado com o metotrexato, a terapia gold standard, e responde bem clinicamente mantendo sua qualidade de vida, como ocorre em muitos casos, não há 
necessidade de trocar este medicamento ou por outro de maior custo (dentre os disponíveis até 2006), pois os dados de literatura indicam que essa troca não irá acrescentar melhora relevante para este paciente. Cabe ao prescritor identificar o paciente não respondedor, o mais rápido possível, e então decidir pelo medicamento com preço superior.

Para melhor tomada de decisão, há necessidade de pesquisas sobre expectativa de efetividade de tratamento de pacientes com artrite reumatóide nas fases iniciais da doença, sem tratamento prévio, e na seqüência de necessidade de alternativas de tratamento. A qualidade das decisões de órgãos ou instituições financiadores de tratamentos dependem dessa informação básica.

Deve haver questionamentos de quanto tratamentos mais caros devam ser usados e para que grupo de pacientes ele é custo-efetivo para ser incluído.

\section{ABSTRACT}

\section{Cost analysis of drug therapy in rheumatoid arthritis}

With the aim to compare the cost of treatment for rheumatoid arthritis therapy with desease-modifying antirheumatic drugs (DMARDs) for a 48-month period, were studied five different treatment stage based on clinical protocols recommended by the Brazilian Society of Rheumatology, and then five therapy cycles. The analytical model based on the Markov Analysis, considered chaces for the patient continue in some stages or change between them according with a positive effect on outcomes. Only direct costs were comprised in the analyzed data, like drugs, materials and tests used for monitoring these patients. The results of the model show that the stage in with metotrexato drug is used like monotherapy was cost-effective ( $R \$ 113,900.00$ for patient during 48 months), followed by refractory patient ( $R \$$ $1,554,483.43)$, those that use therapy triplicate followed by infleximabe drug $(R \$ 1,701,286.76)$, the metotrexato intolerant patient $(R \$ 2,629,919.14)$, and final the result from that use metotrexato and infliximabe in the beginning ( $R \$ 9,292,879.31)$. The sensitivity analysis confirm this results, when alternate the efficacy of metotrexato and infliximabe.

UNITERMS: Rheumatoid arthritis/drug therapy. DMARDs. Anti-TNF. Pharmacoeconomy analysis.

\section{REFERÊNCIAS BIBLIOGRÁFICAS}

CLASSIFICAÇÃO Brasileira Hierarquizada de Procedimentos Médicos. 4.ed. São Paulo: Editora AMB, 2005. 199 p.

DRUMMOND, M.F.; O'BRIEN, B.J.; STODDART, G.L.; TORRANCE, GW. Methods for the economic evaluation of health care programmes. London: Oxford Medical Publications, $1998.200 \mathrm{p}$.

EMERY, P. Review of health economics modeling in rheumatoid arthritis. Pharmacoeconomics, v.22, supl.1, p.55-69, 2004.

GABRIEL, S.E.; TUGWELL, P.; DRUMMOND, M. Progress towards an OMERACT-ILAR guideline for economic evaluations in rheumatology. Ann. Rheum. Dis., v.61, p.370-373, 2002.

GABRIEL, S.E.; COLYLE, D.; MORELAND, L.W. A clinical and economic review of disease-modifying antirheumatic drugs. Pharmacoeconomics, v.19, n.7, p.715-728, 2001.

GUIA Farmacêutico Brasíndice. São Paulo: Andrei Publicações Médicas, 2006. 230 p.

LAURINDO, I.M.M.; PINHEIRO, G.R.C.; XIMENES, A.C.; BERTOLO, M.B.; XAVIER, R.M.; GIORGI, R.D.N.; CICONELLI, R.M.; RADOMINSKI, S.C.; LIMA, F.A.C.; BATISTELA, L.; ALENCAR, P. Consenso brasileiro para o diagnóstico e tratamento da artrite reumatóide. Rev. Bras. Reumatol., v.72, n.6, p.355$361,2002$.

LUBECK, D.P. A review of the direct costs of rheumatoid arthritis. Managed Care versus Fee-for-Service Settings. Pharmacoeconomics, v.19, n.8, p.811-818, 2001.

MARCH, L.; LAPSLEY, H. What are the costs to society and the potential benefits from the effective management of early rheumatoid arthritis? Bailliére 's Best Prac. Res. Clin. Rheumatol., v.15, n.1, p.171-185, 2001.

MERKESDAL, S.; RUOF, J.; HUELSEMANN, J.L.; SCOEFFSKI, O.; MAETZEL, A.; MAU, W.; ZEIDLER, H. Development of a matrix of cost domains in economic evaluation of rheumatoid arthritis. $J$. Rheumatol., v.28, p.657-661, 2001. 
MONTEIRO, R.D.C. Análise de custo do tratamento medicamentoso da artrite reumatóide. São Paulo, 2006. f.151. [Dissertação de Mestrado. Faculdade de Ciências Farmacêuticas. Universidade de São Paulo].

NURMOHAMED, M.T.; DIJKMANS, B.A.C. Efficacy, tolerability and cost effectiveness of disease-modifying antirheumatic drugs and biologic agents in rheumatoid arthritis. Drugs, v.65, n.5, p.661-694, 2005.

OLLENDORF, D.A.; PETERSON, A.N.; DOYLE, J.; HUSE, D.M. Impact of leflunomida versus biologic agents on the costs of care for rheumatoid arthritis in a Managed Care population. Am. J. Manag. Care, v.8, n.7, p.S203-S213, 2002.

POLANEZYK, C.A.; RIBEIRO, J.P. Análise e estimativa dos custos das intervenções médicas. Estudos de CustoEfetividade em Cardiologia: Da Teoria à Prática, São Paulo: Lemos, 1998 n.3, p 12-15.

SCHADLICH, P.K.; ZEIDLER, H.; ZINK, A.; GROMNICA-IHLE, E.; SCHNEIDER, M.; STRAUB, C.; BRECHT, J.G.; HUPPERTZ, E. Modeling cost effectiveness and cost utility of sequential DMARD therapy including leflunomida in rheumatoid arthritis in Germany: I. Selected DMARDs and patient-related costs. Pharmacoeconomics, v.23, n.4, p.377-393, 2005.
SCOTT, D.L. Pursuit of optimal outcomes in rheumatoid arthritis. Pharmacoeconomics, v.22, supl.1, p.13-26, 2004.

SCOTT, D.L.; SHIPLEY, M.; DAWSON, A.; EDWARDS, S.; SYMMONS, D.P.M.; WOOLF, A.D. The clinical management of rheumatoid arthritis and osteoarthritis strategies for improving clinical effectiveness. $\mathrm{Br} . \mathrm{J}$. Rheumatol., v.37, p.546-554, 1998.

SOLOMON, D.H.; AVORN, J., Pharmacoepidemiology and rheumatic diseases: 2001-2002. Curr. Opin. Rheumatol., v.15, n.2, p.122-126, 2003.

TUGWELL, P. Farmacoeconomia da terapia medicamentosa para artrite reumatóide. Rheumatol., v.39, supl.1, p.43-47, 2000 .

ZANINI, A.C.; WADT, M. Farmacoeconomia. In: LOPES, A.C.; AMATO NETO, V. (Eds.) Tratado de clínica médica. São Paulo: Roca, 2006. v.1, cap.38, p.370-378.

Recebido para publicação em 22 de novembro de 2006 Aceito para publicação em 4 de dezembro de 2007 